\title{
Single Amino Acid Substitution in Bacillus sphaericus Phenylalanine Dehydrogenase Dramatically Increases Its Discrimination between Phenylalanine and Tyrosine Substrates
}

\author{
Stephen Y. K. Seah, ${ }^{\ddagger}, \perp$ K. Linda Britton, ${ }^{\S}$ David W. Rice, ${ }^{\S}$ Yasuhisa Asano," and Paul C. Engel ${ }^{*, \hbar}$ \\ Department of Biochemistry and Conway Institute of Biomolecular and Biomedical Research, University College Dublin, \\ Belfield, Dublin 4, Ireland, Krebs Institute for Biomolecular Research, Department of Molecular Biology and Biotechnology, \\ University of Sheffield, P.O. Box 594, Sheffield S10 2UH, United Kingdom, and Biotechnology Research Center, Toyama \\ Prefectural University, 5180 Kurokawa, Kosugi, Toyama 939-03, Japan
}

Received March 12, 2002; Revised Manuscript Received May 20, 2002

\begin{abstract}
Homology-based modeling of phenylalanine dehydrogenases (PheDHs) from various sources, using the structures of homologous enzymes Clostridium symbiosum glutamate dehydrogenase and Bacillus sphaericus leucine dehydrogenase as a guide, revealed that an asparagine residue at position 145 of $B$. sphaericus PheDH was replaced by valine or alanine in PheDHs from other sources. This difference was proposed to be the basis for the poor discrimination by the B. sphaericus enzyme between the substrates L-phenylalanine and L-tyrosine. Residue 145 of this enzyme was altered, by site-specific mutagenesis, to hydrophobic residues alanine, valine, leucine, and isoleucine, respectively. The resultant mutants showed a high discrimination, above 50-fold, between L-phenylalanine and L-tyrosine. This higher specificity toward L-phenylalanine was due to $K_{\mathrm{m}}$ values for L-phenylalanine lowered more than 20-fold compared to the values for L-tyrosine. The greater specificity for L-phenylalanine in the wild-type Bacillus badius enzyme, which has a valine residue in the corresponding position, was also found to be largely due to a lower $K_{\mathrm{m}}$ for this substrate. Activities were also measured with a range of six amino acids with aliphatic, nonpolar side chains, and with the corresponding oxoacids, and in all cases the specificity constants for these substrates were increased in the mutant enzymes. As with phenylalanine, these increases are mainly attributable to large decreases in $K_{\mathrm{m}}$ values.
\end{abstract}

The label phenylalanine dehydrogenase $\left(\mathrm{PheDH}^{1}\right)$ (EC 1.4.1.20) denotes a class of enzymes that catalyze the reversible $\mathrm{NAD}^{+}$-dependent oxidative deamination of a broad range of hydrophobic amino acid substrates, with particular preference for aromatic side chains. The enzyme from Bacillus sphaericus is unusual within this class since its activities toward L-phenylalanine and L-tyrosine are similar $(1,2)$. In contrast, PheDH from Rhodococcus sp. M4 shows 34-fold discrimination in favor of L-phenylalanine (3). Similarly, with the enzymes from Bacillus badius and Sporosarcina ureae, tyrosine gives only 9 and 5.4\%, respectively, of the specific activity with phenylalanine $(1,4)$. It is of interest to understand the structural basis for this difference in discrimination between aromatic amino acid substrates that differ by only a single hydroxyl substituent. Determining the molecular details of substrate recognition in the family of amino acid dehydrogenases has also practical

* Corresponding author. Phone 003531716 1547; Fax 003531 283 7211; E-mail paul.engel@ucd.ie.

$\doteqdot$ University College Dublin.

$\S$ University of Sheffield.

" Toyama Prefectural University.

${ }^{\perp}$ Present address: Department of Microbiology, University of Guelph, Guelph, Ontario, Canada.

1 Abbreviations: PheDH, phenylalanine dehydrogenase; LeuDH, leucine dehydrogenase; GluDH, glutamate dehydrogenase; $\mathrm{NAD}^{+}$, oxidized nicotinamide adenine dinucleotide; NADH, reduced nicotinamide adenine dinucleotide. SDS-PAGE, sodium dodecyl sulfatepolyacrylamide gel electrophoresis. relevance, as these enzymes are useful as biocatalysts for stereospecific synthesis of amino acids, and as diagnostic reagents for a variety of inborn errors of amino acid metabolism. Indeed, the thermostability of PheDH from $B$. sphaericus would recommend its use as a diagnostic reagent. However, major interference from tyrosine makes it unsuitable for measuring serum L-phenylalanine levels in the diagnosis of phenylketonuria (PKU) (2).

The rational design of enzymes with altered substrate specificities for use in chemical synthesis or clinical diagnosis is a major goal of our protein engineering efforts. Several notable examples of successful redesign of substrate specificities depend on prior knowledge of the high-resolution structure of the enzyme, or at least of the corresponding enzyme from another biological species. In the absence of such information, site-directed mutagenesis may be guided by patterns of amino acid conservation, by the results of chemical modification studies, or by molecular modeling if the structure of a related, homologous protein is known. Homology-based modeling has been fruitful, for example, in the redesign of coenzyme specificity in glutathione reductase and lipoamide dehydrogenase $(5,6)$. A critical question remains, however, as to the level of amino acid sequence similarity that might justify such an approach.

Across the family of amino acid dehydrogenases, similarity in terms of amino acid sequence identity is not strikingly high. For instance, the overall sequence identity between 
Clostridium symbiosum glutamate dehydrogenase (GluDH) and B. sphaericus PheDH is only $13 \%$. Nevertheless, molecular modeling based on the sequence alignment and the solved structure of $C$. symbiosum $\operatorname{GluDH}(7,8)$ suggested that the active sites have similar structures (9). Thus, for example, three glycine residues that orient functional groups and determine the basic shape of the active sites are all conserved in GluDH, LeuDH (leucine dehydrogenase), and $\mathrm{PheDH}$. In addition, the lysine residue that interacts with the $\alpha$-carboxyl group of the amino acid substrate, and also aspartate 165 and lysine 125 (GluDH numbering), thought to be involved in the catalytic mechanisms of the enzymes $(8,10,11)$, are also conserved. The modeling study also pointed to differences in a small number of amino acid residues within the active site that govern the substrate specificity of the different classes of enzymes, and this guided site-directed mutagenesis in which the substrate specificity of $B$. sphaericus $\mathrm{PheDH}$ was successfully shifted toward that of leucine dehydrogenase (LeuDH) (12). Mutagenesis of a similar amino acid residue in valine dehydrogenase likewise supports the validity of the modeling study (13).

In this paper, we have taken a similar approach, using the known tertiary structures of $C$. symbiosum GluDH (4) and also B. sphaericus $\mathrm{LeuDH}$ (14) as a guide to align the amino acid sequences of PheDHs from various sources to identify the likely structural basis of differing levels of discrimination between phenylalanine and tyrosine. The resulting hypothesis has been tested by site-directed mutagenesis and detailed kinetic analysis of the mutant enzymes. Our results again suggest that, even when overall amino acid sequence identity is below $20 \%$, precise conclusions may be drawn regarding the functional role of key residues.

The structure of a PheDH, from Rhodococcus sp. M4, has now been directly determined by X-ray crystallography (15), but was not available during the mutational study described in this paper (2). It should be noted also that, even with an authentic structure now available, the basis for phenylalanine and tyrosine discrimination in PheDH has not as yet been addressed.

\section{MATERIALS AND METHODS}

Materials. Restriction enzymes and T4 DNA ligase were from Life Technologies Inc, calf intestinal alkaline phosphatase was from Promega Corp. (UK), and T4 polymerase used in the synthesis of the second strand during mutagenesis was from Cambio. DNA sequencing was performed using the 7-deaza dGTP kit and Sequenase Version 2.0 (United States Biochemicals, USA). The Geneclean II kit was obtained from BIO 101 (La Jolla, CA). Oligonucleotides for mutagenesis were synthesized by Genosys Biotechnologies, Inc. (UK). Oligonucleotides for DNA sequencing were synthesized using the Cyclone Plus DNA synthesizer. Sepharose CL-6B and Butyl Sepharose 4 fast flow were from Pharmacia Biotech (UK). Procion Red P3BN was from Imperial Chemical Industries (UK). Amino acid (free acid) and oxoacid (sodium salts) substrates of greater than 98\% purity were purchased from Sigma Chemical Company (UK). $\mathrm{NAD}^{+}$(Grade II, free acid, 98\% purity) and NADH (Grade I, disodium salt, $100 \%$ purity) were from Boehringer Mannheim (UK).

Sequence Alignment. PheDH sequences were aligned using the structure of $C$. symbiosum $\operatorname{GluDH}(7,16)$ as a reference as previously reported for PheDH of B. sphaericus (9). The additional PheDH sequences, from B. badius (17), S. ureae (18), and Rhodococcus sp. M4 (19) were added to the alignment with the aid of the program CLUSTALW (20).

Construction of Mutants. The uracilated template method $(21,22)$ was employed for mutagenesis on the B. sphaericus $p d h$ gene inserted in the BamHI site of the vector M13mp19. The mutagenic oligonucleotide GAGACGAATTTCATTGCCGGAATTCCTGAG (the underlined codon GCC replacing AAC) was designed to replace asparagine at position 145 with alanine, while the sequence GAGACGAATTTCATTXTCGGAATTCCTGAG (where $\mathrm{X}$ is degenerate, being either $\mathrm{C}, \mathrm{A}$, or $\mathrm{G}$ ) was chosen to replace the same residue with leucine, isoleucine, and valine, respectively. Mutants were screened directly by sequencing using the dideoxychain termination method (23).

Expression and Purification of Wild-Type and Mutant B. sphaericus PheDH. Wild-type and mutant $p d h$ genes were subcloned into the BamHI site of the vector ptac 85 and transformed into Escherichia coli TG1. The correct orientation of the gene with the $5^{\prime}$ end positioned directly downstream of the tac promoter in the vector was confirmed by EcoRI digests, since there are two asymmetrically positioned EcoRI sites within the gene and one within the vector.

The wild-type and mutant $B$. sphaericus enzymes were purified as described previously (12). Purity was monitored by SDS-PAGE (12\%) (24). The concentration of purified $B$. sphaericus $\mathrm{PheDH}$ was determined by absorbance at $280 \mathrm{~nm}$ using an extinction coefficient of $1.17 \times 10^{-3} \mathrm{~g}^{-1}$ $\mathrm{mL} \mathrm{cm}{ }^{-1}$ (4).

Expression and Purification of B. badius Enzyme. The $B$. badius $\mathrm{PheDH}$ enzyme was purified as previously described (4). Concentration of the enzyme was calculated from absorbance at $280 \mathrm{~nm}$ using an extinction coefficient of $6.3 \times 10^{-2} \mathrm{~g}^{-1} \mathrm{~mL} \mathrm{~cm}^{-1}(4)$.

Molecular Weight Determination. The molecular weights of the native enzymes were determined by FPLC gel filtration (LCC-500, Pharmacia) on a Superose 6 HR 10/30 column (Pharmacia) at a flow rate of $0.5 \mathrm{~mL} / \mathrm{min}$. The elution buffer contained $50 \mathrm{mM}$ phosphate buffer, $\mathrm{pH} 7.9$, with $0.15 \mathrm{M} \mathrm{NaCl}$. The column was calibrated with apoferritin $\left(M_{\mathrm{r}}=443000\right)$, C. symbiosum glutamate dehydrogenase $\left(M_{\mathrm{r}}=295000\right)$, sweet potato $\beta$-amylase $\left(M_{\mathrm{r}}=200000\right)$, rabbit muscle aldolase $\left(M_{\mathrm{r}}=158000\right)$, bovine albumin $\left(M_{\mathrm{r}}=68000\right)$, and hen egg albumin $\left(M_{\mathrm{r}}=45000\right)$.

Subunit molecular weights were estimated from a $12 \%$ SDS-PAGE gel with the following molecular weight markers: bovine albumin $\left(M_{\mathrm{r}}=66000\right)$, egg albumin $\left(M_{\mathrm{r}}=45000\right)$, rabbit muscle glyceraldehyde-3-phosphate dehydrogenase $\left(M_{\mathrm{r}}=36000\right)$, bovine erythrocyte carbonic anhydrase $\left(M_{\mathrm{r}}=29000\right)$, bovine pancreas PMSF-treated trypsinogen $\left(M_{\mathrm{r}}=24000\right)$, soybean trypsin inhibitor $\left(M_{\mathrm{r}}=\right.$ $20000)$, and bovine milk $\alpha$-lactalbumin $\left(M_{\mathrm{r}}=14200\right)$.

Enzyme Assays. Oxidative deamination was assayed at $25^{\circ} \mathrm{C}$ by measuring the reduction of $\mathrm{NAD}^{+}$at $340 \mathrm{~nm}$ in a $1-\mathrm{cm}$ cuvette. The $K_{\mathrm{m}}$ values of the mutant enzymes for $\mathrm{NAD}^{+}$were obtained by fixing the concentration of $\mathrm{L}^{-}$ phenylalanine at $1 \mathrm{mM}$ (at least 10-times the apparent $K_{\mathrm{m}}$ in all cases) and varying the $\mathrm{NAD}^{+}$concentration. The reaction mixture also contained $50 \mu \mathrm{mol}$ of glycine- $\mathrm{KOH}$ buffer, $\mathrm{pH} 10.4,100 \mu \mathrm{mol}$ of $\mathrm{KCl}$, and the appropriate amount of 


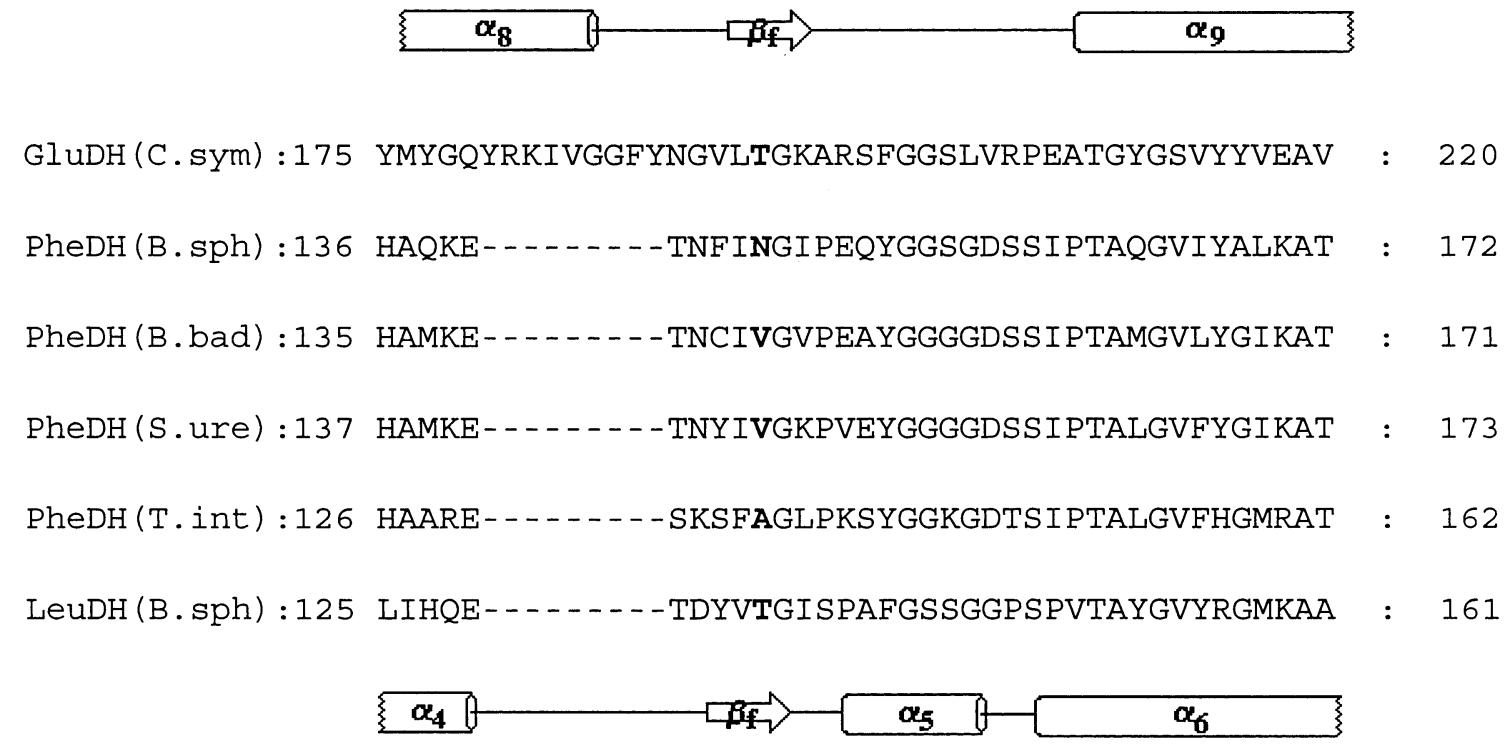

FIGURE 1: Sequence alignment of PheDHs using structural information from $C$. symbiosum GluDH and B. sphaericus LeuDH showing the residue (bold) that is thought to be an important determinant of phenylalanine/tyrosine specificity. Sequences shown are for GluDH from C. symbiosum (C. sym) (16), PheDH from B. sphaericus (B. sph) (27), PheDH from B. badius (B. bad) (17), PheDH from S. ureae (S. ure) (18), PheDH from T. intermedius (T. int) (28), and LeuDH from B. sphaericus (B. sph) (14). The secondary structure of $C$. symbiosum GluDH is indicated above the sequences and that of B. sphaericus LeuDH below. $\alpha$-Helices are indicated as cylinders and $\beta$-strands as arrows. Residue numbers are indicated at both ends of each sequence.

enzymes in a total volume of $1 \mathrm{~mL}$. The true extrapolated $K_{\mathrm{m}}$ value for $\mathrm{NAD}^{+}$in the wild-type enzyme was determined (2) as $\phi_{\mathrm{NAD}^{+}} / \phi_{0}$ from primary and secondary plots (25) of data using varying concentrations of L-phenylalanine and $\mathrm{NAD}^{+}$. To determine kinetic parameters for amino acid substrates, the $\mathrm{NAD}^{+}$concentration was fixed at $2.5 \mathrm{mM}$, and the amino acid concentration was varied.

The disappearance of NADH during reductive amination at $25{ }^{\circ} \mathrm{C}$ was monitored at $340 \mathrm{~nm}$ in a $1-\mathrm{mL}$ mixture containing $50 \mu \mathrm{mol}$ of glycine- $\mathrm{KOH}, 100 \mu \mathrm{mol}$ of $\mathrm{KCl}, 0.1$ $\mu \mathrm{mol}$ of NADH, $400 \mu \mathrm{mol}$ of ammonium chloride, varying amounts of oxoacids, and appropriate amounts of enzyme at a $\mathrm{pH}$ of 8.6.

The range of concentrations of each amino acid or oxoacid substrate was at least 10-fold, with a minimum of three different concentrations both above and below the $K_{\mathrm{m}}$ value. One unit of enzyme was the amount that catalyzed the formation or disappearance of $1 \mu \mathrm{mol}$ of $\mathrm{NADH} / \mathrm{min}$. Steadystate kinetic parameters, $K_{\mathrm{m}}$ and $k_{\text {cat }}$, were obtained by fitting data from duplicate activity readings to the MichaelisMenten equation using the method of Wilkinson (26) in the program Enzpack version 3.0 (Biosoft)

\section{RESULTS}

Three-Dimensional Modeling and Sequence Alignment. Multiple sequence alignment of PheDHs, using the structure of $C$. symbiosum GluDH as a guide, reveals the presence of 10 residues in PheDHs of B. badius, Thermoactinomyces intermedius, and $S$. ureae that are replaced with chemically distinct residues at the corresponding amino acid positions in the $B$. sphaericus enzyme (Table 1). In considering which of these residues might account for the varying degree of discrimination among PheDHs toward phenylalanine and tyrosine as substrates, molecular modeling drew our attention to a critically placed asparagine residue at position 145 in B. sphaericus. In GluDH and LeuDH, a strictly conserved threonine residue is found at the corresponding position
Table 1: Comparison of Amino Acid Residues in PheDH of $B$ sphaericus that Are Replaced with Chemically Distinct Residues in the PheDHs of B. badius, $S$. ureae, and T. intermedius. Amino Acid Positions Are Based on the B. sphaericus PheDH Sequence

\begin{tabular}{ccccc}
\hline $\begin{array}{c}\text { amino acid } \\
\text { position }\end{array}$ & B. sphaericus & B. badius & S. ureae & T. intermedius \\
\hline 54 & $\mathrm{~T}$ & $\mathrm{C}$ & $\mathrm{C}$ & $\mathrm{C}$ \\
74 & $\mathrm{E}$ & $\mathrm{K}$ & $\mathrm{K}$ & $\mathrm{K}$ \\
105 & $\mathrm{~A}$ & $\mathrm{E}$ & $\mathrm{E}$ & $\mathrm{E}$ \\
138 & $\mathrm{Q}$ & $\mathrm{M}$ & $\mathrm{M}$ & $\mathrm{A}$ \\
145 & $\mathrm{~N}$ & $\mathrm{~V}$ & $\mathrm{~V}$ & $\mathrm{~A}$ \\
262 & $\mathrm{~K}$ & $\mathrm{E}$ & $\mathrm{D}$ & $\mathrm{D}$ \\
281 & $\mathrm{~K}$ & $\mathrm{~L}$ & $\mathrm{~A}$ & $\mathrm{~V}$ \\
286 & $\mathrm{~A}$ & $\mathrm{G}$ & $\mathrm{G}$ & $\mathrm{G}$ \\
354 & $\mathrm{~K}$ & $\mathrm{M}$ & $\mathrm{M}$ & $\mathrm{I}$ \\
358 & $\mathrm{~T}$ & $\mathrm{~A}$ & $\mathrm{~A}$ & $\mathrm{~A}$ \\
\hline
\end{tabular}

(Figure 1). In the three-dimensional structures of both $C$. symbiosum GluDH and B. sphaericus LeuDH, this threonine is close to the terminus of the amino acid substrate side chain. In $\mathrm{GluDH}$, this residue is involved in hydrogen bonding with lysine 89 and serine 380, which in turn interact with the $\gamma$-carboxyl group of the glutamate substrate (6). In LeuDHs (Figure 2) and PheDHs, both K89 and S380 are replaced by hydrophobic residues, leucine and valine, respectively, which have been shown, using the GluDH framework, to be important for accommodating hydrophobic substrates $(29,30)$. The $\gamma$-carbon of the threonine residue in $B$. sphaericus LeuDH is within $5 \AA$ of the $\delta$-carbon of a modeled leucine substrate (Figure 2) (31). In PheDHs, the larger aromatic substrate would be much closer to the corresponding residue, which is valine in B. badius and $S$. ureae and alanine in $T$. intermedius (Figure 1). PheDH from Rhodococcus sp. M4 (not shown) displays much lower overall sequence similarity but nevertheless also has a hydrophobic residue, phenylalanine, in this position, corresponding with its high substrate preference for phenylalanine over tyrosine. Asparagine at this position has in fact been found so far only in B. sphaericus, and modeling suggests 


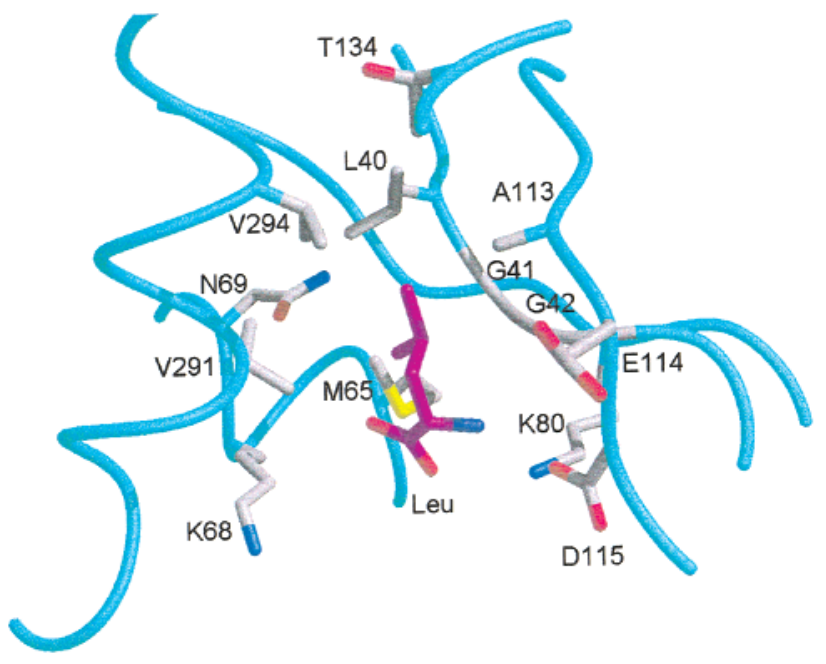

FIGURE 2: Structure of the substrate binding pocket of LeuDH from B. sphaericus (14) showing the positions of residue threonine 134 and the $\delta$-carbon of the modeled leucine substrate. Drawn with MIDASPLUS $(38,39)$.

that the amide group of asparagine 145 may provide relatively favorable possibilities for interaction with the hydroxyl group of a tyrosine substrate and less so for the nonpolar phenyl ring. Mutations of asparagine 145 in $B$. sphaericus $\mathrm{PheDH}$ to the aliphatic nonpolar amino acids, alanine, valine, leucine, and isoleucine were therefore made to determine the contribution of these residues to substrate specificity.

Mutagenesis. The four site-directed mutations N145A, N145L, N145I, and N145V were successfully made by the Kunkel method, and in each case the entire DNA sequence was checked to confirm that no secondary mutations had occurred. This check was repeated after subcloning into the expression vector (by subcloning back into ptac85).

Purification and Analysis of Molecular Weight of Mutant Enzymes. The four mutant enzymes were all overexpressed in good yield and in a soluble form by following the standard procedure for the wild-type enzyme. Wild-type and mutant B. sphaericus $\mathrm{PheDH}$ proteins were purified to more than $99 \%$ homogeneity as judged from a $12 \%$ SDS-PAGE. All five enzymes showed the same mobility on SDS-PAGE, and the subunit molecular weights were estimated to be 39 $\mathrm{kDa}$. In FPLC gel filtration experiments, the retention times of the native mutant enzymes were the same as for the wildtype, indicating an apparent native molecular weight of 380 $\mathrm{kDa}$, and showing that the various substitutions of asparagine 145 do not disrupt the native quaternary structure.

$K_{m}$ for Coenzyme $N A D^{+}$. The true extrapolated $K_{\mathrm{m}}$ for $\mathrm{NAD}^{+}$for the wild type $B$. sphaericus $\mathrm{PheDH}$ is $0.24 \mathrm{mM}$ (12). The apparent $K_{\mathrm{m}}$ values for $\mathrm{NAD}^{+}$in the mutants were estimated by using a fixed L-phenylalanine concentration of $1 \mathrm{mM}$. Since this is more than 20 times the apparent wildtype $K_{\mathrm{m}}$ value for this substrate, these apparent $K_{\mathrm{m}}$ values are likely to be very close to the true extrapolated values. The values obtained ranged from $0.19 \mathrm{mM}$ for N145I and $0.21 \mathrm{mM}$ for N145A to $0.26 \mathrm{mM}$ for both $\mathrm{N} 145 \mathrm{~V}$ and N145L. The fact that these are so similar to the wild-type value suggests that the mutations do not greatly affect coenzyme binding.

Kinetic Parameters for Aromatic Substrates. $K_{\mathrm{m}}$ and $k_{\mathrm{cat}}$ values for the aromatic substrates in the oxidative deami-
Table 2: Kinetic Parameters of Wild-Type and Mutant $B$. sphaericus and Wild-Type B. badius PheDH Enzymes for Aromatic Substrates $^{a}$

\begin{tabular}{clccc}
\hline & & & & \\
substrate & enzyme & $K_{\mathrm{m}}(\mathrm{mM})$ & $k_{\text {cat }}\left(\mathrm{s}^{-1}\right)$ & $\left(\mathrm{s}^{-1} \mathrm{mM}^{-1}\right)$ \\
\hline L-phenylalanine & wt & $1.1 \pm 0.070$ & $68 \pm 1.4$ & 62 \\
& N145A & $0.042 \pm 0.0035$ & $34 \pm 0.94$ & 809 \\
& N145V & $0.021 \pm 0.0031$ & $17 \pm 0.97$ & 810 \\
& N145I & $0.014 \pm 0.00088$ & $13 \pm 0.30$ & 929 \\
& N145L & $0.032 \pm 0.0031$ & $8.5 \pm 0.28$ & 266 \\
& B. badius & $0.087 \pm 0.0087$ & $39 \pm 1.5$ & 448 \\
L-tyrosine & wt & $1.3 \pm 0.10$ & $68 \pm 2.1$ & 52 \\
& N145A & $2.0 \pm 0.21$ & $31 \pm 1.3$ & 15 \\
& N145V & $1.2 \pm 0.065$ & $23 \pm 0.42$ & 19 \\
& N145I & $1.3 \pm 0.13$ & $21 \pm 0.81$ & 16 \\
& N145L & $0.69 \pm 0.049$ & $9.4 \pm 0.22$ & 14 \\
& B. badius & $8.6 \pm 0.78$ & $34 \pm 1.6$ & 3.9 \\
phenylpyruvate & wt & $0.37 \pm 0.035$ & $113 \pm 5.3$ & 305 \\
& N145A & $0.030 \pm 0.026$ & $71 \pm 2.0$ & 2367 \\
& N145V & $0.025 \pm 0.0019$ & $60 \pm 1.3$ & 2400 \\
& N145I & $0.012 \pm 0.0013$ & $43 \pm 1.5$ & 3583 \\
& N145L & $0.0095 \pm 0.00075$ & $23 \pm 0.46$ & 2421 \\
& wt & $0.19 \pm 0.016$ & $90 \pm 2.8$ & 474 \\
p-hydroxy- & N145A & $0.099 \pm 0.0076$ & $14 \pm 0.38$ & 141 \\
phenylpyruvate & N145V & $0.61 \pm 0.083$ & $88 \pm 4.6$ & 144 \\
& N145I & $0.55 \pm 0.035$ & $80 \pm 2.1$ & 145 \\
& N145L & $0.22 \pm 0.021$ & $30 \pm 1.1$ & 136 \\
\hline
\end{tabular}

${ }^{a}$ Kinetic parameters for the oxo-acid substrates in the reductive amination reactions for the $B$. badius enzyme were not determined.

Table 3: Discrimination between Hydroxylated and Non-Hydroxylated Substrates in $\mathrm{PheDH}^{a}$

\begin{tabular}{lcc}
\hline \multicolumn{1}{c}{ enzymes } & $\begin{array}{c}\text { phenylalanine: } \\
\text { tyrosine }\end{array}$ & $\begin{array}{c}\text { phenylpyruvate: } \\
\text { hydroxyphenylpyruvate }\end{array}$ \\
\hline B. sphaericus WT & 1.2 & 0.64 \\
N145A & 54 & 16.8 \\
N145V & 43 & 16.7 \\
N145I & 58 & 24.7 \\
N145L & 19 & 17.8 \\
B. badius WT & 115 & ND \\
\hline
\end{tabular}

${ }^{a} \mathrm{ND}$ : not determined. The figures given for each form of PheDH are the ratios between the values of $k_{\text {cat }} / K_{\mathrm{m}}$ for the non-hydroxylated substrate and the corresponding hydroxylated substrate.

nation and reductive amination reactions are presented in Table 2. Briefly, substitutions of asparagine 145 (N145) by aliphatic nonpolar amino acids increase the specificity constant $\left(k_{\text {cat }} / K_{\mathrm{m}}\right)$ for L-phenylalanine 8 - to 12 -fold and that for phenylpyruvate in the reverse reaction 4- to 15 -fold relative to the wild type. At the same time, specificity constants for L-tyrosine and phenylhydroxypyruvate are lowered about 3-fold in both cases, thus resulting in a large overall increase in the effective discrimination between the hydroxylated and non-hydroxylated substrates. Table 3 shows the ratio of specificity constants for the two aromatic substrates in the oxidative deamination and reductive amination reactions. The highest discrimination achieved between phenylalanine and tyrosine (specificity constant phenylalaninel specificity constant $t_{\text {tyrosine}}$ ) is over 50 -fold, seen in the mutants N145A and N145I, as compared with only 1.2-fold in wild type $B$. sphaericus PheDH. The mutant N145I also shows the highest discrimination factor in the reductive amination reaction, 25-fold, as compared with 0.64-fold in the wildtype enzyme (which shows a slight preference for $p$ hydroxyphenylpyruvate, mainly due to a favorable $K_{\mathrm{m}}$ value). The overall improvement in the discrimination factor in 
Table 4: Kinetic Parameters for Aliphatic Amino Acids in $B$. sphaericus PheDHs

\begin{tabular}{|c|c|c|c|c|}
\hline substrates & enzyme & $K_{\mathrm{m}}(\mathrm{mM})$ & $k_{\text {cat }}\left(\mathrm{s}^{-1}\right)$ & $\begin{array}{c}k_{\mathrm{cat}} / K_{\mathrm{m}} \\
\left(\mathrm{s}^{-1} \mathrm{mM}^{-1}\right)\end{array}$ \\
\hline L-leucine & $\begin{array}{l}\text { wt } \\
\text { N145A } \\
\text { N145V } \\
\text { N145I } \\
\text { N145L }\end{array}$ & $\begin{array}{c}0.55 \pm 0.066 \\
0.032 \pm 0.0015 \\
0.013 \pm 0.0013 \\
0.015 \pm 0.0020 \\
0.021 \pm 0.0017\end{array}$ & $\begin{aligned} 0.70 & \pm 0.026 \\
0.98 & \pm 0.012 \\
0.84 & \pm 0.030 \\
1.2 & \pm 0.056 \\
0.45 & \pm 0.0098\end{aligned}$ & $\begin{array}{l}1.3 \\
31 \\
65 \\
80 \\
21\end{array}$ \\
\hline L-isoleucine & $\begin{array}{l}\text { wt } \\
\text { N145A } \\
\text { N145V } \\
\text { N145I } \\
\text { N145L }\end{array}$ & $\begin{aligned} 0.14 & \pm 0.023 \\
0.017 & \pm 0.0011 \\
0.0095 & \pm 0.00077 \\
0.014 & \pm 0.00097 \\
0.0082 & \pm 0.00091\end{aligned}$ & $\begin{array}{l}0.14 \pm 0.009 \\
0.50 \pm 0.012 \\
0.15 \pm 0.0034 \\
0.19 \pm 0.0033 \\
0.11 \pm 0.0039\end{array}$ & $\begin{array}{l}1.0 \\
29 \\
16 \\
14 \\
13\end{array}$ \\
\hline L-norleucine & $\begin{array}{l}\text { wt } \\
\text { N145A } \\
\text { N145V } \\
\text { N145I } \\
\text { N145L }\end{array}$ & $\begin{array}{c}0.79 \pm 0.069 \\
0.031 \pm 0.0021 \\
0.074 \pm 0.0088 \\
0.052 \pm 0.0041 \\
0.056 \pm 0.0039\end{array}$ & $\begin{array}{l}3.2 \pm 0.054 \\
3.4 \pm 0.070 \\
5.6 \pm 0.21 \\
5.4 \pm 0.17 \\
3.3 \pm 0.060\end{array}$ & $\begin{array}{r}4.0 \\
110 \\
76 \\
104 \\
59\end{array}$ \\
\hline L-methionine & $\begin{array}{l}\text { wt } \\
\text { N145A } \\
\text { N145V } \\
\text { N145I } \\
\text { N145L }\end{array}$ & $\begin{aligned} 2.7 & \pm 0.19 \\
0.078 & \pm 0.0055 \\
0.13 & \pm 0.0090 \\
0.14 & \pm 0.0043 \\
0.15 & \pm 0.015\end{aligned}$ & $\begin{array}{l}1.2 \pm 0.034 \\
3.1 \pm 0.085 \\
2.9 \pm 0.083 \\
3.1 \pm 0.036 \\
1.8 \pm 0.064\end{array}$ & $\begin{array}{l}0.44 \\
40 \\
22 \\
22 \\
12\end{array}$ \\
\hline L-norvaline & $\begin{array}{l}\text { wt } \\
\text { N145A } \\
\text { N145V } \\
\text { N145I } \\
\text { N145L }\end{array}$ & $\begin{array}{c}3.3 \pm 0.22 \\
0.11 \pm 0.014 \\
0.18 \pm 0.018 \\
0.14 \pm 0.014 \\
0.11 \pm 0.0089\end{array}$ & $\begin{array}{c}1.4 \pm 0.038 \\
1.8 \pm 0.11 \\
1.9 \pm 0.077 \\
2.0 \pm 0.078 \\
0.91 \pm 0.026\end{array}$ & $\begin{array}{l}0.42 \\
16 \\
11 \\
14 \\
8.3\end{array}$ \\
\hline L-valine & $\begin{array}{l}\text { wt } \\
\text { N145A } \\
\text { N145V } \\
\text { N145I } \\
\text { N145L }\end{array}$ & $\begin{array}{c}2.0 \pm 0.10 \\
0.14 \pm 0.015 \\
0.27 \pm 0.047 \\
0.23 \pm 0.032 \\
0.27 \pm 0.037\end{array}$ & $\begin{array}{c}0.91 \pm 0.020 \\
0.85 \pm 0.034 \\
1.4 \pm 0.10 \\
1.6 \pm 0.074 \\
0.62 \pm 0.034\end{array}$ & $\begin{array}{l}0.46 \\
6.1 \\
5.2 \\
7.0 \\
2.3\end{array}$ \\
\hline
\end{tabular}

N145I PheDH is thus 48-fold in the oxidative deamination reaction and 39-fold for reductive amination.

Detailed inspection of the $K_{\mathrm{m}}$ values shows that in the wild-type B. sphaericus enzyme, the values are similar for L-phenylalanine $(1.1 \mathrm{mM})$ and L-tyrosine $(1.3 \mathrm{mM})$. However, in the mutants, the $K_{\mathrm{m}}$ for L-phenylalanine is at least 20-fold lower than for L-tyrosine, with a maximum difference of 93-fold seen in the mutant N145I $(0.014 \mathrm{mM}$ for L-phenylalanine and $1.3 \mathrm{mM}$ for L-tyrosine). $k_{\text {cat }}$ values for the two substrates in each mutant are, however, surprisingly similar.

To ascertain whether the higher specificity for L-phenylalanine in PheDHs of other organisms is also due to a lower $K_{\mathrm{m}}$ value compared to that for L-tyrosine, the kinetic parameters for the $B$. badius enzyme, which has a valine corresponding to position 145 of the $B$. sphaericus enzyme, were determined (Table 2). The results were qualitatively rather similar to those obtained for the $B$. sphaericus mutants, and it can be seen that the $K_{\mathrm{m}}$ for L-phenylalanine is indeed 100 -fold lower than that for L-tyrosine, while the two $k_{\text {cat }}$ values are very similar. $K_{\mathrm{m}}$ values, however, were somewhat higher for both substrates with the B. badius enzyme, 2-6fold for L-phenylalanine, and 4-12-fold for L-tyrosine. The values for the N145A mutant are closest to those found for the wild-type $B$. badius enzyme.

Kinetic Parameters for Aliphatic Substrates. Kinetic parameters for six aliphatic hydrophobic amino acids and the corresponding oxo-acids are shown in Table 4 and Table 5. Specificity constants for all these substrates are much increased in the mutant enzymes (Table 6), and these
Table 5: Kinetic Parameters for Aliphatic Oxoacids in $B$. sphaericus $\mathrm{PheDHs}^{a}$

\begin{tabular}{|c|c|c|c|c|}
\hline substrates & enzyme & $K_{\mathrm{m}}(\mathrm{mM})$ & $k_{\text {cat }}\left(\mathrm{s}^{-1}\right)$ & $\begin{array}{c}k_{\mathrm{cat}} / K_{\mathrm{m}} \\
\left(\mathrm{s}^{-1} \mathrm{mM}^{-1}\right)\end{array}$ \\
\hline$\alpha$-ketoisocaproate & $\begin{array}{l}\text { wt } \\
\text { N145A } \\
\text { N145V } \\
\text { N145I } \\
\text { N145L }\end{array}$ & $\begin{array}{c}20 \pm 2.7 \\
0.22 \pm 0.019 \\
0.21 \pm 0.013 \\
0.21 \pm 0.013 \\
0.22 \pm 0.017\end{array}$ & $\begin{array}{l}27 \pm 2.0 \\
24 \pm 0.72 \\
18 \pm 0.37 \\
28 \pm 0.55 \\
15 \pm 0.37\end{array}$ & $\begin{array}{c}1.4 \\
109 \\
86 \\
133 \\
68\end{array}$ \\
\hline $\begin{array}{l}\text { DL- } \alpha \text {-keto- } \beta \text { - } \\
\text { methyl- } n \text {-valerate }\end{array}$ & $\begin{array}{l}\text { wt } \\
\text { N145A } \\
\text { N145V } \\
\text { N145I } \\
\text { N145L }\end{array}$ & $\begin{aligned} 13 & \pm 1.8 \\
0.74 & \pm 0.067 \\
0.76 & \pm 0.071 \\
1.0 & \pm 0.069 \\
1.6 & \pm 0.15\end{aligned}$ & $\begin{aligned} 7.2 & \pm 0.40 \\
19 & \pm 0.52 \\
9.6 & \pm 0.27 \\
16 & \pm 0.34 \\
13 & \pm 0.42\end{aligned}$ & $\begin{array}{l}0.55 \\
26 \\
13 \\
16 \\
8.1\end{array}$ \\
\hline$\alpha$-ketocaproate & $\begin{array}{l}\text { wt } \\
\text { N145A } \\
\text { N145V } \\
\text { N145I } \\
\text { N145L }\end{array}$ & $\begin{aligned} 13 & \pm 0.70 \\
0.25 & \pm 0.018 \\
0.15 & \pm 0.012 \\
0.25 & \pm 0.019 \\
0.22 & \pm 0.017\end{aligned}$ & $\begin{array}{l}51 \pm 1.3 \\
66 \pm 1.6 \\
40 \pm 1.1 \\
61 \pm 1.8 \\
42 \pm 1.2\end{array}$ & $\begin{array}{l}3.9 \\
264 \\
267 \\
244 \\
191\end{array}$ \\
\hline $\begin{array}{l}\alpha \text {-keto- } \gamma \text {-methio- } \\
\text { butyrate }\end{array}$ & $\begin{array}{l}\text { wt } \\
\text { N145A } \\
\text { N145V } \\
\text { N145I } \\
\text { N145L }\end{array}$ & $\begin{array}{c}11 \pm 0.73 \\
0.26 \pm 0.018 \\
0.29 \pm 0.011 \\
0.43 \pm 0.032 \\
0.28 \pm 0.024\end{array}$ & $\begin{array}{l}27 \pm 0.78 \\
61 \pm 1.5 \\
34 \pm 0.044 \\
47 \pm 1.2 \\
25 \pm 0.78\end{array}$ & $\begin{array}{l}3.7 \\
235 \\
117 \\
109 \\
89\end{array}$ \\
\hline$\alpha$-ketovalerate & $\begin{array}{l}\text { wt } \\
\text { N145A } \\
\text { N145V } \\
\text { N145I } \\
\text { N145L }\end{array}$ & $\begin{array}{l}52 \pm 4.4 \\
1.4 \pm 0.060 \\
2.2 \pm 0.070 \\
2.6 \pm 0.26 \\
2.9 \pm 0.18\end{array}$ & $\begin{array}{l}33 \pm 1.8 \\
54 \pm 0.78 \\
40 \pm 0.47 \\
61 \pm 2.4 \\
40 \pm 1.0\end{array}$ & $\begin{array}{l}0.63 \\
39 \\
18 \\
23 \\
14\end{array}$ \\
\hline$\alpha$-ketoisovalerate & $\begin{array}{l}\text { wt } \\
\text { N145A } \\
\text { N145V } \\
\text { N145I } \\
\text { N145L }\end{array}$ & $\begin{array}{l}\text { ND } \\
3.3 \pm 0.45 \\
4.2 \pm 0.16 \\
4.3 \pm 0.17 \\
7.4 \pm 0.50\end{array}$ & $\begin{array}{l}\text { ND } \\
54 \pm 0.78 \\
46 \pm 0.72 \\
59 \pm 0.98 \\
36 \pm 1.0\end{array}$ & $\begin{array}{l}0.55^{*} \\
16 \\
11 \\
14 \\
4.9\end{array}$ \\
\hline
\end{tabular}

${ }^{a} \mathrm{ND}: K_{\mathrm{m}}$ too high to be determined. *: determined from the gradient of a specific activity vs substrate concentration curve.

Table 6: Improvement in Performance of Mutant PheDHs with Aliphatic Substrates $^{a}$

\begin{tabular}{lcccc}
\hline \multicolumn{1}{c}{ substrates } & $\mathrm{N} 145 \mathrm{~A}$ & $\mathrm{~N} 145 \mathrm{~V}$ & $\mathrm{~N} 145 \mathrm{I}$ & $\mathrm{N} 145 \mathrm{~L}$ \\
\hline leucine & 23.8 & 50.0 & 61.5 & 16.2 \\
isoleucine & 29.0 & 16.0 & 14.0 & 13.0 \\
norleucine & 27.5 & 19.0 & 26.0 & 14.8 \\
methionine & 90.9 & 50.0 & 50.0 & 27.3 \\
norvaline & 38.1 & 26.2 & 33.3 & 19.8 \\
valine & 13.3 & 11.3 & 15.2 & 5.0 \\
$\alpha$-ketoisocaproate & 77.9 & 61.4 & 95.0 & 48.6 \\
$\alpha$-keto- $\beta$-methyl- $n$-valerate & 47.3 & 23.6 & 29.1 & 14.7 \\
$\alpha$-ketocaproate & 67.7 & 68.5 & 62.6 & 49.0 \\
$\alpha$-keto- $\gamma$-methiobutyrate & 63.5 & 31.6 & 29.5 & 24.1 \\
$\alpha$-ketovalerate & 61.9 & 28.6 & 36.5 & 22.2 \\
$\alpha$-ketoisovalerate & 29.1 & 20.0 & 25.5 & 8.9 \\
\hline
\end{tabular}

${ }^{a}$ In each case, the figure given is the ratio, for a particular substrate, of the specificity constants of the mutant enzyme and the wild type. Thus, for example, with L-methionine, the specificity constants for $\mathrm{N} 145 \mathrm{~V}$ and wild-type are 22 and $0.44 \mathrm{~s}^{-1} \mathrm{mM}^{-1}$, respectively. The corresponding ratio, given in the table, is 50 .

increases, as for the aromatic substrates, are mainly attributable to reduction in $K_{\mathrm{m}}$ values. The largest increase in specificity constant, 91 -fold relative to the wild-type, is seen with methionine as substrate for the N145A mutant $\left(40 \mathrm{~s}^{-1}\right.$ $\mathrm{mM}^{-1}$ compared to $0.44 \mathrm{~s}^{-1} \mathrm{mM}^{-1}$ in the wild-type). Norleucine is also a reasonably good substrate for all four mutants; although the highest $k_{\mathrm{cat}}$ figure of $5.6 \mathrm{~s}^{-1}(\mathrm{~N} 145 \mathrm{~V})$ is much lower than most of the corresponding figures for either L-phenylalanine or L-tyrosine, the low $K_{\mathrm{m}}$ values for norleucine result in specificity constants for three of the 
mutants well in excess of the wild-type figure of $62 \mathrm{~s}^{-1}$ $\mathrm{mM}^{-1}$ for L-phenylalanine.

\section{DISCUSSION}

Substitution of asparagine 145 in B. sphaericus $\mathrm{PheDH}$ by aliphatic hydrophobic amino acids has resulted in enzymes with greatly increased discrimination between the substrates L-phenylalanine and L-tyrosine. The $k_{\text {cat }}$ values are similar for both substrates with each mutant, but for all four mutants, the $K_{\mathrm{m}}$ values for L-phenylalanine are substantially lower than that for L-tyrosine, with factors of up to 93-fold, as observed in the N145I mutant. In PheDH from B. badius, which has a valine corresponding to N145 of B. sphaericus PheDH, $K_{\mathrm{m}}$ for L-phenylalanine $(0.088 \mathrm{mM})$ is also much lower than for L-tyrosine ( $8.6 \mathrm{mM}$ ) (Table 2). As with the N145 mutants, the $k_{\text {cat }}$ values for both substrates in the B. badius enzyme are similar. Reproduction of this trend in the mutants confirms that the residue at position 145 determines the differential specificity among the PheDH enzymes toward L-phenylalanine and L-tyrosine as substrates.

The dominant influence of alterations in $K_{\mathrm{m}}$ in determining the effective substrate preference inevitably raises a question as to the physical significance of the $K_{\mathrm{m}}$ for the amino acid substrate in this case. Michaelis constants are frequently loosely taken as an indication of binding affinity, but for a number of mechanisms this identification is unjustified. For PheDH under these conditions, however, our evidence so far (2) points to a rapid-equilibrium random-order mechanism. If this is indeed the mechanism, the $K_{\mathrm{m}}$ for each substrate can be identified with the $K_{\mathrm{d}}$ for its dissociation, not from the corresponding binary complex, but from the ternary complex (32). This would then imply that N145 and its various replacements, either natural or engineered, control the tightness of binding of L-phenylalanine and L-tyrosine to the E-NAD ${ }^{+}$complex, but that tight binding does not guarantee optimal orientation for catalysis, which is presumably ensured by other interactions with the aromatic ring and $\alpha$-carbon substituents.

The obvious possible factors bearing upon the discrimination between phenylalanine and tyrosine as substrates include steric hindrance, hydrogen bonding, and hydrophobic or van der Waals interactions. Steric exclusion of tyrosine, due to the presence of the $p$-hydroxyl group, has been suggested as the basis for the preference for L-phenylalanine in E. coli phenylalanyl-tRNA synthetase (33). Substitution of alanine 294 by a smaller glycine resulted in an enzyme able to activate tyrosine and form an enzyme-tyrosyl-AMP complex, unlike the wild-type enzyme. Steric hindrance is an inadequate explanation in the present case, however, since the same effect is seen even when the asparagine residue is replaced by a smaller alanine in the N145A mutant. Moreover, the enhanced discrimination results mainly from improved binding of phenylalanine rather than hindered binding of tyrosine.

The second possibility to consider is the loss of a putative hydrogen bond between asparagine 145 and the hydroxyl group of tyrosine. The contributions of hydrogen bonding in Bacillus stearothermophilus tyrosyl-tRNA synthetase have been previously explored, a context where the ability to distinguish between phenylalanine and tyrosine (150 000fold in favor of tyrosine in this case) is biologically critical (34). The crucial distinction between absolute energy of formation of a single hydrogen bond and the overall net energy of the hydrogen bonding process in solution is pointed out in that study. It is the overall energy change that drives the binding of a substrate to an enzyme. For example, in the absence of substrate, tyrosine 34 and aspartate 176 in tyrosyl tRNA synthetase form hydrogen bonds with enzyme-bound water. Likewise, the tyrosine substrate in solution forms hydrogen bonds with water molecules. When the substrate binds to the enzyme, hydrogen bonding between Y34 and D176 of the enzyme and the tyrosine substrate must compensate for the loss of hydrogen bonding to the displaced water. Substitution of tyrosine 34 by phenylalanine, which breaks one of the hydrogen bonding interactions with the substrate resulted in a 15-fold decrease in the selectivity for tyrosine (34). Likewise, it is possible that, upon binding of the tyrosine substrate in $B$. sphaericus $\mathrm{PheDH}$, new hydrogen bonding interactions in the bulk solvent and of asparagine with the substrate might similarly compensate for the loss of the original hydrogen bonds, perhaps explaining the similar specificity constants observed for L-phenylalanine and L-tyrosine in the wild-type enzyme. In the N145 mutants, the loss of hydrogen bonding between tyrosine and water could no longer be compensated by hydrogen bonding between substrate and the amide group of N145, and substantially weakened binding of tyrosine should result. There is indeed approximately a 3-fold diminution in $k_{\mathrm{cat}} / K_{\mathrm{m}}$ for tyrosine in the mutants (Table 2), but this results mainly from decreased values for $k_{\text {cat }}$ rather than increases in $K_{\mathrm{m}}$ and would in any case not explain the more than 90-fold difference in specificity constants for L-phenylalanine and L-tyrosine seen in some of the mutants.

The increase in specificity for phenylalanine can, however, be reasonably explained in part by the increased hydrophobicity of the active site and in part by van der Waals interaction between the substituent in the N145 position and the substrate. This would also be consistent with the increase in specificity constants for a variety of hydrophobic aliphatic substrates in the mutants, which is again mainly attributable to decreased $K_{\mathrm{m}}$ values (Tables 4 and 5). Conversely, the diminution in $k_{\text {cat }}$ for L-tyrosine seen in the mutants could reflect a suboptimal orientation enforced by the unfavorable interaction of the extra hydroxyl group with an increasingly hydrophobic environment. An interesting comparison can be made between these PheDH mutants with an aspartate-tovaline mutation (D425V) of tyrosine hydroxylase (35). Replacement of a carboxylate with a methyl group in residue 425 of tyrosine hydroxylase also results in a significant decrease (80-fold) in $K_{\mathrm{m}}$ for phenylalanine substrate.

The differences in specificity for different aliphatic substrates in the various N145 mutants can be explained by the balance of two opposing factors, hydrophobicity and steric effects, which are likely to play a more important role in the acceptance or rejection of these substrates than with tyrosine. Hydrophobicity of an amino acid is proportional to its surface area (36), and therefore the substitutes for N145 in the mutants increase in hydrophobicity in the order alanine $>$ valine > leucine, isoleucine. However, steric hindrance of substrate binding also increases in that order. Therefore, in the comparison of the N145V and N145A mutants, for example, the higher hydrophobicity of valine compared to alanine results in tighter binding of the substrate phenylalanine $\left(K_{\mathrm{m}}=0.021 \mathrm{mM}\right.$ for $\mathrm{N} 145 \mathrm{~V}$ compared with 0.042 
$\mathrm{mM}$ for $\mathrm{N} 145 \mathrm{~A})$, but nevertheless not so productively ( $k_{\mathrm{cat}}$ for $\mathrm{N} 145 \mathrm{~V}=17 \mathrm{~s}^{-1}$ compared to $34 \mathrm{~s}^{-1}$ for N145A). Steric hindrance in $\mathrm{N} 145 \mathrm{~V}$ probably prevents phenylalanine from binding in the catalytically optimal orientation. Comparing the mutants N145I and N145L, it appears that the $\gamma$-branch in leucine makes a larger contribution to steric hindrance compared to the $\beta$-branch in isoleucine, although the side chains are of equal size. This is evident from the generally lower specificity constants of N145L for most of the substrates in both the oxidative deamination and reductive amination reactions.

The largest increase in specificity constant is seen with the substrate methionine in the N145A. The long side chain of methionine is probably sterically hindered by residues larger than alanine, explaining lower specificity constants seen in the other mutants. For leucine, isoleucine, and norleucine and their corresponding oxo-acids, the relative trends in the values of the specificity constants are still the same as in the wild type (norleucine $>$ leucine $>$ isoleucine and ketocaproate $>$ ketoisocaproate $>$ keto-methyl- $n$-valerate) (Tables 4 and 5). This suggests that shape features of the active site affecting differential substrate specificity are preserved in these mutants. The wild-type enzyme has similar specificity constants for valine $\left(0.46 \mathrm{~s}^{-1} \mathrm{mM}\right)$ and norvaline $\left(0.42 \mathrm{~s}^{-1} \mathrm{mM}\right)$ and also for the corresponding oxoacids $\alpha$-ketoisovalerate $\left(0.55 \mathrm{~s}^{-1} \mathrm{mM}\right)$ and $\alpha$-ketovalerate $(0.63$ $\left.\mathrm{s}^{-1} \mathrm{mM}\right)$. However, in the mutants the specificity constants for straight-chain norvaline and $\alpha$-ketovalerate are higher than for branched valine and $\alpha$-ketoisovalerate, respectively, possibly because the flexible straight chain allows optimal hydrophobic interaction.

The fact that substitution of asparagine 145 increases the specificity constants for all aliphatic hydrophobic amino acids suggests that this residue could also be one of the sequence differences between PheDH and LeuDH that determine the differing specificity of these two enzymes. Previous analysis (12) has highlighted the importance of Gly 124 and Leu 307 (in PheDH) in this regard. In all LeuDHs, however, the residue corresponding to Asn 145 is threonine, which has a $\beta$-branch and, though polar, is less hydrophilic than asparagine. Since the mutants with a $\beta$-branch, N145V and N145I, perform better with aliphatic substrates than the mutant with a $\gamma$-branch, N145L, it appears that steric effects are significant here.

In the practical application of these enzymes for PKU diagnosis, a low $K_{\mathrm{m}}$ value for L-phenylalanine is an advantage. The levels of L-phenylalanine and L-tyrosine in the serum of normal individuals are about $0.06 \mathrm{mM}$ (37). At these concentrations, the activity of the mutant enzymes toward L-phenylalanine would be slightly below the maximum catalytic rate $\left(K_{\mathrm{m}}\right.$ between 0.01 and $\left.0.04 \mathrm{mM}\right)$, but specific activities for L-tyrosine would be far below $k_{\text {cat }}\left(K_{\mathrm{m}}\right.$ between 0.69 to $2.01 \mathrm{mM}$ ). Individuals with PKU have elevated levels of serum L-phenylalanine, up to $2.4 \mathrm{mM}$, and therefore, interference from L-tyrosine would be further reduced. The mutational modification of $B$. sphaericus $\mathrm{PheDH}$ to produce a diagnostic tool suitable for PKU testing provides a successful example of the rational design of altered substrate specificity. In particular, this study demonstrates the potential of sequence alignment and homologybased modeling for predicting mutagenic targets when no direct 3D structures are available.
Although the present study was based on homology modeling, without the benefit of a directly determined 3D structure for PheDH, the X-ray crystal structure for PheDH from Rhodococcus sp. M4 has recently been reported (15). This enzyme has a high preference for phenylalanine over tyrosine, and it also possesses a hydrophobic residue, phenylalanine (F138), corresponding to the Asn 145 position of $B$. sphaericus PheDH. Our examination of the published structure for the enzyme-substrate complex of Rhodococcus PheDH reveals that F138 is at the base of the active-site pocket, about $4 \AA$ away from the L-phenylalanine (or other) substrate. This is consistent with our conclusion that hydrophobicity in the interior of the binding pocket has a major bearing on phenylalanine/tyrosine discrimination in this class of enzymes. It should, however, be pointed out that, whereas the PheDH's of B. sphaericus, B. badius, S. ureae, and T. intermedius share at least $49 \%$ amino acid identities, the enzyme from Rhodococcus shows only $27 \%$ identity with these others (1). Specifically, some of the residue differences in the Rhodococcus enzyme map at the substrate binding site. For example, all the other PheDHs listed have a leucine residue corresponding to position 51 of the B. sphaericus enzyme, but this residue is replaced with alanine in the Rhodococcus enzyme. This residue is about $4 \AA$ away both from F138 and from the bound substrate. The smaller alanine residue at this position may be important in accommodating the large F138 side chain and may also have some influence on the overall substrate specificity.

The availability of a solved high-resolution structure for PheDH should in theory provide a much less speculative starting point for mutagenesis. Clearly, however, the sequence differences mentioned above would diminish the certainty of predictions for the $B$. sphaericus enzyme on which we have worked. While evidently it should now be possible to apply the detailed knowledge of Rhodococcus PheDH to relatively confident mutagenesis for that specific enzyme, the real message of the work described here is that, even without the benefit of a 3D structure directly determined for the enzyme in question, well-guided homology modeling may still permit a precise and successful outcome to sitedirected mutagenesis.

\section{NOTE ADDED AFTER ASAP POSTING}

This article appeared ASAP on August 30, 2002 with an incorrect phenylalanine residue cited in the penultimate paragraph of the text. The correct residue (F138) appears in this version posted September 24, 2002.

\section{REFERENCES}

1. Asano, Y., Nakazawa, A., and Endo, K. (1987) J. Biol. Chem. 262, 10346-10354.

2. Seah, S. Y. K. (1997) Ph.D. Thesis, University of Sheffield

3. Hummel, W., Schütte, H., Schmidt, E., Wandrey, C., and Kula, M.-R. (1987) Appl. Microbiol. Biotechnol. 26, 409-416.

4. Asano, Y., Nakazawa, A., Endo, K., Hibino, Y., Ohmori, M., Numao, N., and Kondo, K. (1987) Eur. J. Biochem. 168, 153159.

5. Scrutton, N. S., Berry, A., and Perham, R. N. (1990) Nature 343, $39-43$.

6. Bocanegra, J. A., Scrutton, N. S., and Perham, R. N. (1993) Biochemistry 23, 2737-2740.

7. Baker, P. J., Britton, K. L., Engel, P. C., Farrants, G. W., Lilley, K. S., Rice, D. W., and Stillman, T. J. (1992) Proteins: Struct., Funct., Genet. 12, 75-86. 
8. Stillman, T. J., Baker, P. J., Britton, K. L., and Rice, D. W. (1993) J. Mol. Biol. 234, 1131-1139.

9. Britton, K. L., Baker, P. J., Engel, P. C., Rice, D. W., and Stillman, T. J. (1993) J. Mol. Biol. 234, 938-945.

10. Dean, J. L. E., Wang, X.-G., Teller, J. K., Waugh, M. L., Britton, K. L., Baker, P. J., Stillman, T. J., Martin, S. R., Rice, D. W., and Engel, P. C. (1994) Biochem. J. 201, 13-16.

11. Brunhuber, N. M., Thoden, J. B., Blanchard, J. S., and Vanhooke, J. L. (2000) Biochemistry 39, 9174-9187.

12. Seah, S. Y. K., Britton, K. L., Baker, P. J., Rice, D. W., Asano, Y., and Engel, P. C. (1995) FEBS Lett. 370, 93-96.

13. Hyun, C. G., Kim, S. S., Lee I. H., and Suh, J. W. (2000) Antonie Van Leeuwenhoek 78, 237-242.

14. Baker, P. J., Turnbull, A. P., Sedelnikova, S. E., Stillman, T. J., and Rice, D. W. (1995) Structure 3, 693-705.

15. Vanhooke, J. L., Thoden, J. B., Brunhuber, N. M., Blanchard, J. S., and Holden, H. M. (1999) Biochemistry 38, 2326-2339.

16. Teller, J. K, Smith, R. J., McPherson, M. J., Engel, P. C., and Guest, J. R. (1992) Eur. J. Biochem. 206, 151-159.

17. Yamada, A., Dairi, T., Ohno, Y., Huang, X. L., and Asano, Y. (1995) Biosci. Biotechnol. Biochem. 59, 1994-1995.

18. Asano, Y. Genbank accession number AB001031.

19. Brunhuber, N. M., Banerjee, A., Jacobs, W. R., Jr., and Blanchard, J. S. (1994) J. Biol. Chem. 269, 16203-16211.

20. Thompson, J. D., Higgins, D. G., and Gibson, T. J. (1994) Nucleic Acids Res. 22, 4673-4680.

21. Kunkel, T. A. (1985) Proc. Natl. Acad. Sci. U.S.A. 82, 488-492.

22. Kunkel, T. A., Roberts, J. D., and Zakour, R. A. (1987) Methods Enzymol. 154, 367-382.

23. Sanger, F., Nicklen, S., and Coulson, A. R. (1977) Proc. Natl. Acad. Sci. U.S.A. 74, 5463-5467.

24. Laemmli, U. K. (1970) Nature 227, 680-685.

25. Dalziel, K. (1957). Acta Chem. Scand. 11, 1706-1723.
26. Wilkinson, G. N. (1961) Biochem. J. 80, 324-332.

27. Okazaki, N., Hibino, Y., Asano, Y., Ohmori, M., Numao, N., and Kondo, K. (1988) Gene 63, 337-34

28. Takada, H., Yoshimura, T., Ohshima, T., Esaki, N., and Soda, K. (1991) J. Biochem. 109, 371-376.

29. Stillman, T. J., Migueis, A. M., Wang, X.-G., Baker, P. J., Britton, K. L., Engel, P. C., and Rice, D. W. (1999) J. Mol. Biol. 285, 875-885.

30. Wang, X.-G., Britton, K. L., Stillman, T. J., Rice, D. W., and Engel, P. C. (2001) Eur. J. Biochem. 268, 5791-5799.

31. Turnbull, A. P., Baker, P. J., and Rice, D. W. (1997) J. Biol. Chem. 272, 25105-25111.

32. Engel, P. C. (1981) Enzymes: The Steady-State Approach, 2nd ed., Chapman and Hall, London.

33. Ibba, M., Kast, P., and Hennecke, H. (1994) Biochemistry 33, 7107-7112.

34. Fersht, A. R., Shi, J.-P., Knill-Jones, J., Lowe, D. M., Wilkinson, A. J., Blow, D. M., Brick, P., Carter, P., Waye, M. M. Y., and Winter, G. (1985) Nature 314, 235-238.

35. Dauber, S. C., Melendez, J., and Fitzpatrick, P. F. (2000) Biochemistry 39, 9652-9661.

36. Rose, G. D., Geselowitz, A. R., Lesser, G. J., Lee, R. H., and Zehfus, M. H. (1985) Science 229, 834-838.

37. Hummel, W., Schütte, H., and Kula, M.-R. (1988). Anal. Biochem. 170, 397-401.

38. Ferrin, T. E., Huang, C. C., Jarvis, L. E., and Langridge, R. (1988) J. Mol. Graphics 6, 13-27.

39. Huang, C. C., Pettersen, E. F., Klein, T. E., Ferrin, T. E., and Langridge, R. (1991) J. Mol. Graphics 9, 230-236.

BI020196A 